\title{
Effects of a Protocol for the Preparation of Oxygen-Moisturizing Chambers on the Count of Its Bacteria Colony
}

\author{
Reyhaneh Alikhani $^{1}$, Mohsen Shahriari ${ }^{2} \&$ Sina Mobasherizadeh ${ }^{3}$ \\ ${ }^{1}$ School of Nursing and Midwifery, Isfahan University of Medical Sciences, Isfahan, Iran \\ ${ }^{2}$ Nursing and Midwifery Care Research Center, School of Nursing and Midwifery, Isfahan University of \\ Medical Sciences, Hezarjrib Ave., Isfahan, Iran \\ ${ }^{3}$ Nosocomial Infection Research Center, Isfahan University of Medical Sciences, Iran \\ Correspondence: Mohsen Shahriari, Nursing \& Midwifery Care Research Center, School of Nursing and \\ Midwifery, Isfahan University of Medical Sciences, Hezarjrb Ave., Isfahan, Iran. Tel: 98-31-3792-7516. Fax: \\ 98-31-3669-9398. E-mail: shahriari@nm.mui.ac.ir
}

Received: May 3, 2016 Accepted: June 16, 2016 Online Published: June 30, 2016

doi:10.5539/gjhs.v9n2p160 URL: http://dx.doi.org/10.5539/gjhs.v9n2p160

\begin{abstract}
This study aims to evaluate the effect of the protocol for the preparation of oxygen-moisturizing chamber on the count of its bacteria.

This study is a double- group clinical trial before and after intervention, which was carried out in the neurology ward of an educational hospital in Esfahan from July to December 2015. In this study, 64 oxygen humidifying chambers were studied in terms of number and type of microorganisms in two groups of 32 during three phases, before, immediately after, and 6 hours after its intervention. In order to identify the microorganisms, the samples were cultured on the blood agar and EMB (Eosin Methylene Blue). Then, the routine laboratory methods were used to identify the types of microorganisms. Data were analyzed using SPSS 18, Wilcoxon, Mann-Whitney, chi-square and Friedman statistical tests.

The results showed that before intervention, $71.9 \%$ (46/64) of oxygen-moisturizing chambers were contaminated with microorganisms. The extent of microbial contamination was from 0 to $10^{5} \mathrm{CFU}$. Most of the contamination was with microorganisms such as Lactobacillus spp 23.4\% (15/64), Bacillus sp17.2\% (11/64), Pseudomonas aeruginosa 10.9\% (7/64), Coagulase-negative staphylococci 7.8\% (5/64), Acinetobacter baumannii 3.1\% (2/64), Sphingomonas spp 3.1\%(2/64), Escherichia coli 1.6\% (1/64), Streptococcus spp 3.1\% (2/64), Fungus spp 3.1\% $(2 / 64)$. However, immediately after the intervention and 6 hours after connecting oxygen-moisturizing chamber to the patient, the infection rate was substantially reduced and reached zero $(\mathrm{p}<0 / 001)$.

With regard to the fact that the contamination of oxygen-moisturizing chamber was significantly reduced after the implementation of care protocol, the implementation of this Protocol can be one of the effective measures in reducing the transmission of nosocomial infections.
\end{abstract}

Keywords: oxygen, oxygen-moisturizing chamber, microbial count

\section{Introduction}

Nosocomial infections have always been one of the major problems, which have imposed heavy costs on the health care system (Hajibagheri \& Afrasiabian, 2006), in health care centers since the past decades. These infections are an important cause of mortality worldwide, which are annually responsible for 42 to 98 thousand deaths in the United States of America, and between 17 and 19 million dollars are allocated for controlling these infections (Nguyêñ et al., 2000) each year. The incidence of nosocomial infections have been reported to be more than 25\% in Iran (Abdollahi et al., 2003). They could lead to death, permanent complications, increased length duration of hospitalization, increased healthcare costs and dissatisfaction of patients and their entourage. In spite of this, a significant proportion of these infections are preventable (Medina et al., 1996).

One of the most important nosocomial infections is that of the respiratory system, which includes about 15 to 20 percent of nosocomial infections (Brooks, 2001). Respiratory system infection is created and transmitted in different ways, one of which is through providing patients with oxygen. Water tanks are connected to the device 
in order to moisten the oxygen; however, these water reservoirs may be contaminated with germs and may transfer the germs to patients by suspended particles (Sanner, Fluerenbrock, \& Kleiber, 2001; Taheri \& Jokar, 2007).

The study conducted by Rood Dehghan et al. in 2006 showed that the performance of all nurses before the oxygen therapy, with regard to the correctness equipment and cleaning water-oxygen tanks in two conducted visits, was $0 \%$ and weak since only $14.8 \%$ of nurses had used sterile distilled water in the moisturizing chamber of oxygen (Rood-dehghan et al., 2011). Findings of Brokaksiet al. titled errors and mistakes during oxygen therapy of patient hospitalized in Greece showed that 88 percent of nurses stated that there were no protocols for oxygen therapy in their wards and special errors and mistakes had been made in prescriptions, controlling, cleaning, monitoring and cutting oxygen therapy (Brand, 2010). A study carried out by Aslani et al. in 2009 revealed that out of 24 samples prepared from oxygen monometers, $17.5 \%$ had microbial contamination (Aslani et al., 2009). Moreover, the study conducted by Olia et al. in Tehran in 2003 revealed that out of 130 water samples taken from oxygen-moisturizing chambers, $90 \%$ had microbial contamination (Owlia et al., 2001).

Existence of sufficient knowledge and awareness about the direct and indirect ways of transmission of infection-creating agents is considered one of the essential factors in providing daily care for patients. Since providing oxygen is a fundamental activity in nursing practice and nurses are responsible for safe care of patients, existence of adequate knowledge and awareness enables nurses to have an important role in prevention, detection, treatment and restricting the spread of infectious diseases, especially those transmitted from oxygen-moisturizing chambers (Luckman \& Sorensen,1987; Bergogne-Berezin, 1995; Seigel \& Romo, 1990).

Therefore, in this study, efforts have been made to study the impact of care protocol for the preparation of researcher-made oxygen-moisturizing chamber on the bacterial count in oxygen-moisturizing chambers.

\section{Materials and Methods}

\subsection{Sampling, Separation, Diagnosis and Determination of Colony Count of Microorganisms}

This study is of the three-stage double-group clinical trial type. The study population consisted of oxygen-moisturizing chambers in the hospital, which was prepared from neurology wards of one of the educational hospitals in Esfahan for 6 months from July to December 2015. Culture samples were selected from 64 oxygen-moisturizing chambers in the internal and neurosurgery wards, which were randomly assigned in two groups of 32, control and test groups totally from 64 oxygen-moisturizing chambers. 160 water samples were collected by the researcher during two stages, before connecting to the oxygen device and 6 hours after the connection to the oxygen device, and were immediately taken to the laboratory by the very same research. The samples were selected from normal oxygen-moisturizing chambers that had the ability to separate and rinse. In the stage before intervention, Oxygen-moisturizing chambers were filled with sterile water up to the point marked, and then water was given contact to all surfaces within the chamber by swab, after that, $5 \mathrm{ml}$ of this water was taken with a sterile syringe and was poured into capped sterile tubes, and were immediately transported to the laboratory.

In the 32 chambers of the test group, samples were collected in three stages before, immediately after and 6 hours after the implementation of the protocol, which were attached to the oxygen device, and were transported to the laboratory (Owlia et al., 2001). In the 32 oxygen-moisturizing chambers of the control group, no intervention was done before and 6 hours after the chambers were connected to the patient, and the samples were collected using the same method and they were immediately sent to the laboratory.

Water samples sent to the laboratory were centrifuged at $3000 \mathrm{rpm}$ for 10 minutes, and then 10 micro liters of sediments were taken in capped pipes sampled by the laboratory expert with a calibrated loop and were cultured on the blood agar and EMB medium. Culture media were incubated at 35 to $37^{\circ} \mathrm{C}$ for 24 to 72 hours, and then they were evaluated for microorganism growth. After that, the isolated microorganisms were identified by conventional microbiological methods including gram stain, catalase, urease, oxidase, MR, VP tests. Finally, by considering the dilution coefficient of 100 , the number of colonies was counted and recorded. The obtained data were analyzed using statistical-descriptive and analytical software SPSS version 18 and Wilcoxon, Mann-Whitney, chi-square and Friedman statistical tests.

\section{Results}

In the present study, out of 160 water samples taken from oxygen-moisturizing chambers, 64 samples are related to before the implementation of the care protocol phase. Out of these 46 chambers, $71.9 \%$ (46.64) were contaminated with microorganisms, and the range of microbial contamination was from 0 to $10^{5} \mathrm{CFU} / \mathrm{ml}$ (Table 1). The average rates of microorganisms in the oxygen-moisturizing chambers were $26588 \mathrm{CFU} / \mathrm{ml}$ and 0 
$\mathrm{CFU} / \mathrm{ml}$ in before and after intervention respectively (Table 1 ). The most contaminations with microorganisms were Lactobacillus spp 23.4\% (15/64), Bacillus spp 17.2\% (11/64), Pseudomonas. aeruginosa 10.9\% (7/64), Coagulase-negative staphylococci 7.8\% (5/64), Acinetobacter baumannii 3.1\% (2/64), Sphingomonas spp 3.1\% (2/64), Escherichia coli 1.6\% (1/64), Streptococcus spp 3.1\% (2/64), and Fungus spp 3.1\% (2/64), (Table 2). The most common microbial agents that cause respiratory infections in patients were Pseudomonas aeruginosa, Acinetobacter baumannii and Escherichia coli, which were also identified and isolated in the present study (Connie, Donald, \& George, 2011).

However, in the samples that were taken and collected immediately after the intervention and 6 hours after connecting the oxygen-moisturizing chamber to the patient, the infection rate was substantially reduced and reached zero $(\mathrm{p}<0 / 001)$.

Table 1. Mean standard deviation and median of the number of colonies of bacteria in the oxygen-moisturizing chamber in the control and test groups before, time zero and 6 hours after intervention

\begin{tabular}{|c|c|c|c|c|c|}
\hline \multirow[b]{2}{*}{ Statistical index } & \multicolumn{2}{|l|}{ Control } & \multicolumn{3}{|l|}{ Test } \\
\hline & $\begin{array}{l}\text { Before study } \\
\text { (CFU/ml) }\end{array}$ & $\begin{array}{l}6 \text { hours after } \\
\text { study }(\mathrm{CFU} / \mathrm{ml})\end{array}$ & $\begin{array}{l}\text { Before } \\
\text { intervention } \\
(\mathrm{CFU} / \mathrm{ml})\end{array}$ & $\begin{array}{l}\text { Time } \\
\text { zero } \\
(\text { CFU/ml) }\end{array}$ & $\begin{array}{l}6 \text { hours after } \\
\text { intervention }(\mathrm{CFU} / \mathrm{ml})\end{array}$ \\
\hline Mean & 25612 & 25769 & 26588 & 0 & 0 \\
\hline $\begin{array}{l}\text { Standard } \\
\text { deviation }\end{array}$ & 41188 & 41156 & 40157 & 0 & 0 \\
\hline Median & 5500 & 5500 & 6500 & 0 & 0 \\
\hline
\end{tabular}

The results in Table 1 showed that no significant difference was noticed in the test and control groups before intervention as far as the number of microorganisms in the oxygen-moisturizing chamber was concerned $(\mathrm{p}=0.29)$. Nor was any significant difference noticed between the number of microorganisms present in the oxygen-moisturizing chamber before the study and 6 hours after attaching the chambers with oxygen in the control group ( $\mathrm{p}=0.65)$. After the implementation of care protocol (time zero) and even 6 hours after attaching the oxygen-moisturizing chamber to the oxygen, the intensity of microbial contamination was greatly reduced and became zero $(\mathrm{p}<0.001)$.

Table 2. Distribution of bacteria in the oxygen-moisturizing chamber in test and control groups before the intervention

\begin{tabular}{lllllll}
\hline \multirow{2}{*}{ Type of bacteria } & \multicolumn{2}{c}{ Control } & \multicolumn{2}{l}{ Test (Before intervention) } & \multicolumn{2}{c}{ Total } \\
\cline { 2 - 8 } & No & $\mathbf{\%}$ & No & \% & No & \% \\
\hline Coagulase negative Staphylococci & 4 & 12.5 & 1 & 3.1 & 5 & 7.8 \\
Bacillus spp & 6 & 18.8 & 5 & 15.6 & 11 & 17.2 \\
P. aueroginosa & 2 & 6.2 & 5 & 15.6 & 7 & 10.9 \\
Sphingomonas spp & 2 & 6.2 & 0 & 0 & 2 & 3.1 \\
Acinetobacter baumannii & 2 & 6.2 & 0 & 0 & 2 & 3.1 \\
Lactobacillus spp & 7 & 21.9 & 8 & 25 & 15 & 23.4 \\
Streptococcus sp. & 0 & 0 & 1 & 3.1 & 1 & 1.6 \\
Escherichia. coli & 0 & 0 & 1 & 3.1 & 1 & 1.6 \\
Fungus spp & 0 & 0 & 2 & 6.2 & 2 & 3.1 \\
No grow & 9 & 28.1 & 9 & 28.1 & 18 & 28.1 \\
Total & 32 & 100 & 32 & 100 & 62 & 100 \\
\hline
\end{tabular}

As shown in Table 2 regarding the frequency distribution of bacteria and with regard to the fact that there was a quite random selection of samples and at every stage, sampling had been taken from oxygen-moisturizing chamber only once, no significant difference was observed between the test and control groups before intervention in terms of number microorganisms $(\mathrm{p}=0.11)$.In the control group, and 6 hours after the chambers 
were attached to the oxygen, the same number of bacteria was observed with insignificant difference in bacterial count, which was not put in the above Table. However, in the test group, no bacteria were found immediately after the implementation of the protocol and 6 hours after the connection to the patient.

\section{Discussion}

One of the important ways of transmission of microorganisms to patients is the oxygenation process. Water tanks connected to the device are used to moisturize the oxygen. These water tanks may be contaminated with microorganisms and transfer the microorganisms to patients in the form of suspended particles (Sanner, Fluerenbrock, \& Kleiber, 2001; Taheri \& Jokar, 2007). In the current study, the microbial contamination of oxygen-moisturizing chamber was studied. The highest microbial contamination was related to Lactobacillus spp 23.4\% (15/64), Bacillus 17.2\% (11/64), Pseudomonas aeruginosa 10.9\% (7/64), Coagulase-negative staphylococci 7.8\% (5/64). From among bacterial factors, Staphylococci, Enterococci and Pseudomonas aeruginosa have been introduced as the most important organisms that contaminated medical equipment and many internal and external surfaces of many hospital wards (Culver et al., 1991; Edmond \& Wenzel, 1995; Pena et al., 2003). In the studied conducted by Yoosefi et al., most of contaminations were the Bacillus spp (23.1), coagulase negative staphylococci (19.2) and coagulase positive staphylococci (17.6), which is in agreement with the results of the current study, and slight differences in the type of bacteria have been related to the sampling of various medical equipment in addition to the oxygen manometers (Aslani et al., 2009). In the study carried out by Jadhav et al. on oxygen-moisturizing chambers in different wards in a hospital in India in 2011, 61\% microbial contamination, $39 \%$ fungal contamination, $42 \%$ gram negative cocci and $19 \%$ gram positive cocci were extracted (Jadhav et al., 2012). Various studies conducted regarding the identification of microbial factors in oxygen-moisturizing chambers causing respiratory infection, it was shown that microbial factors, which could cause respiratory infection in early days of hospitalization were Streptococci, Staphylococci, Haemophilus influenza and Moraxella catarrhalis. Factors causing respiratory infection 5 to 6 days after hospitalization were Pseudomonas spp and Acinetobacter spp, and after 10 days of hospitalization were Enterobacteriaceae and Pseudomonas aeruginosa (Phillips \& Spencer, 1965; Ringrose et al., 1968; Gandham et al., 2012).

The presence of microorganisms such as Pseudomonas spp and Enterobacteriaceae in the recent study is indicative of lack of adequate washing even in past 10 days, and different types of microorganisms identified in various studies from the present research result from difference in sampling wards.

After the implementation of the care protocol for the preparation of oxygen-moisturizing chambers, it was evident that the microbial contamination of these chambers had approached zero. After initial sampling from oxygen-moisturizing chambers and determination of microbial and fungal contamination, Jadhav et al, too, washed the chambers with water and soap and then disinfected them with ethanol $70 \%$ and used distilled water to fill them. After the disinfection, they took samples again and noticed that the fungal contamination had reached $15 \%$ and microbial contamination had reached 12\% (Jadhav et al., 2012). Considering the fact that high-level disinfecting materials have not been used in the study conducted by Jadhav et al, the difference in microbial contamination observed in both studies is justifiable. With regard to various studies conducted by researchers from 1979 to 2011, the necessity to use distilled water, change it quickly and even use disposable moisturizing chambers in order to decrease the colonization of contaminating bacteria was studied and confirmed. To meet this necessity, this study, too, has been considerably effective in decreasing the microbial contamination transmitted from these chambers by implementing the care protocol for the preparation of moisturizing chambers.

\section{Conclusion}

With regard to the moisture in the oxygen chambers and lack of adequate cleaning, these chambers provide favorable conditions for microbial growth. Providing care protocol for the preparation of these chambers could prevent the formation and colonization of microorganisms. Therefore, it could greatly reduce the secondary costs of treatment of diseases caused by the microbial contamination transferred to patients from these chambers, and increase patients' satisfaction in hospital cares. Hence, it is necessary to pave the way for better implementation of this strategy and care protocol in hospitals by establishing constant control and supervisory systems, securing financial and human resources, and the required physical space.

Considering time constraints, microbial sampling tests were not performed from oxygen-moisturizing chambers at 12,24, 48 and 72 hours after the implementation of care protocol so that maximum useful time or re-implementation time is determined for this protocol for these chambers. Therefore, further supplementary studies should be carried out in a larger time span so that supplementary results are offered to the medical community for the implementation of this care protocol. 


\section{Acknowledgements}

The authors are grateful to Office of Vice-president for Academics and Research of Isfahan University of Medical Sciences for the financial support. Thanks are also extended to the laboratory of Saadi Hospital of Esfahan and Beh Ban Shimi Pharmaceutical Company for their cooperation in implanting this study.

\section{Competing Interests Statement}

The authors declare that there is no conflict of interests regarding the publication of this paper.

\section{References}

Abdollahi, E. A., Rahmani, A. H., Khodabakhshi, B., \& Behnampour, N. (2003). Determine the knowledge, attitude and practice of nurses of Golestan University of Medical Sciences in control of nosocomial infections. J Gorgan Uni Med Sci, 5(11), 80-86. (In Persian). Retrieved from http://www.sid.ir/fa/ VEWSSID/J_pdf/3000113900901.pdf

Aslani, Y., Saadat, M., Etemadifar, S., \& Fazeli, S. M. (2009). The evaluation of different hospital equipment microbial contamination in medical training center Hajar of Shahrekord. Scien J Ham Nurs \& Mid Facul, 17(12), 19-29. (In Persian). Retrieved from http://nmj.umsha.ac.ir/browse.php?a_code=A-10-11060\&sid $=1 \&$ slc_lang=en

Bergogne-Berezin, E. (1995). Nosocomial infections: New agents, incidence, prevention. Presse medicale (Paris, France: 1983), 24(2), 89-97.

Brand, M. D. (2010). The sites and topology of mitochondrial superoxide production. Exp Gerontol, 45(7), 466-472. http://dx.doi.org/10.1016/j.exger.2010.01.003

Brooks, J. A. (2001). Postoperative nosocomial pneumonia: Nurse-sensitive interventions. AACN Advanced Critical Care, 12(2), 305-323. PMID: 11759557. http://dx.doi.org/10.1097/00044067-200105000-00013

Culver, D. H., Horan, T. C., Gaynes, R. P., Martone, W. J., Jarvis, W. R., Emori, T. G., ... Hughes, J. M. (1991). Surgical wound infection rates by wound class, operative procedure, and patient risk index. The Am J Med, 91(3), S152-S157. http://dx.doi.org/10.1016/0002-9343(91)90361-Z

Edmond, M. B., \& Wenzel, R. P. (1995). Nosocomial infections. In G. L. Mandell, J. E. Bennet, \& R. Dolin (Eds.), Principles and practice of infections disease (3rd ed., pp. 2572-2585). New York: Chrchill Livingstone.

Gandham, N. R., Gupta, N., Jadhav, S. V., \& Misra, R. N. (2012). Isolation of Acinetobacter baumannii from Cerebrospinal Fluid following craniotomy. Med J Dr. DY Patil Univ, 5(2), 151. http://dx.doi.org/10. 4103/0975-2870.103347

Hajibagheri, K., \& Afrasiabian, S. (2006). An epidemiologic study of nosocomial infections and its related factors at the intensive care unit of Tohid Hospital. Sanandaj during 2003-2004. Scien J Kurd Univ Med Scien, 44-50. (In Persian). Retrieved from http://sjku.hbi.ir/browse.php?a_code=A-10-1-179 \&slc_lang $=$ fa\&sid $=$ en

Jadhav, S., Gandham, N., Paul, R., Mishra, R. N., Ujagare, M. T., Angadi, K., \& Vyawahare, C. (2012). Bacteriological profile of Septicaemia and antimicrobial Susceptibility of isolates from Tertiary care hospital in India. Res J Pharm Biol Che Sci, 3(4), 1100.

Luckmann, J., \& Sorensen, K. C. (1987). Medical-surgical nursing: A psychophysiologic approach. Saunders.

Mahon, C. R., Lehman, D. C., \& Manuselis, G. (2011). Text Book of Diagnostic Microbiology.upper and lower Respiratory tract infection, 770-804. Retrieved from http://trove.nla.gov.au/version/43015359

Medina, M., Martinez-Gallego, G., Sillero-Arenas, M., \& Delgado-Rodriguez, M. (1996). Risk factors and length of stay attributable to hospital infections of the urinary tract in general surgery patients. Enferm Infecc Microbiol Clin., 15(6), 310-314. PMID: 9376402.

Nguyêñ, G. T., Proctor, S. E., Sinkowitz-Cochran, R. L., Garrett, D. O., \& Jarvis, W. R. (2000). Status of infection surveillance and control programs in the United States, 1992-1996. Am J Infect Control, 28(6), 392-400. PMID: 11114608. http://dx.doi.org/10.1067/mic.2000.110298

Owlia, P., Jalali Nadoushan, M. R., Momenizadeh, A., \& Mohammadian, M. (2001). Contamination of oxygen humidifier reservoirs in hospitals of Tehran. Respiration, 68, 483-7. (In Persian). Retrieved from http://hamkelasy.com/files/pdfarticles/pezeshki/57113820210_(www.hamkelasy.com).pdf

Pena, C., Dominguez, M. A., Pujol, M., Verdaguer, R., Gudiol, F., \& Ariza, J. (2003). An outbreak of 
carbapenem-resistant Pseudomonas aeruginosa in a urology ward. Clin Microbiol Infect, 9(9), 938-943. PMID: 14616682. http://dx.doi.org/10.1046/j.1469-0691.2003.00686.x

Phillips, I., \& Spencer, G. (1965). Pseudomonas aeruginosa cross-infection: Due to contaminated respiratory apparatus. Lancet, 286(7426), 1325-1327. PMID: 4165308. http://dx.doi.org/10.1016/S0140-6736 (65)92344-5

Ringrose, R. E., McKown, B., Felton, F. G., Barclay, B. O., Muchmore, H. G., \& Rhoades, E. R. (1968). A hospital outbreak of Serratia marcescens associated with ultrasonic nebulizers. Ann Intern Med., 69(4), 719-729. PMID: 4878810. http://dx.doi.org/10.7326/0003-4819-69-4-719

Rood-dehghan, Z., Shaban, M., Memari, A., \& Mehran, A. (2011). Staff nurses' adherence to oxygen therapy standards. Iran J Nurs, 23(68), 8-18. (In Persian). Retrieved from http://ijn.iums.ac.ir/browse. php?a_code $=$ A-10-317-3\&slc_lang $=$ en\&sid $=1$

Sanner, B. M., Fluerenbrock, N., Kleiber-Imbeck, A., Mueller, J. B., \& Zidek, W. (2001). Effect of continuous positive airway pressure therapy on infectious complications in patients with obstructive sleep apnea syndrome. Respiration, 68(5), 483-487. PMID: 11939616. http://dx.doi.org/10.1159/000050555

Seigel, D., \& Romo, B. (1990). Extended use of prefilled humidifier reservoirs and the likelihood of contamination. Respiratory care, 35(8), 806-806. PMID: 10145318.

Taheri, Z., \& Jokar, F. (2007). Comparison of knowledge of students and nursing staff in relation to hospital infections. J Infec Dis Trop Med, 1(37), 83-6. (In Persian).

\section{Copyrights}

Copyright for this article is retained by the author(s), with first publication rights granted to the journal.

This is an open-access article distributed under the terms and conditions of the Creative Commons Attribution license (http://creativecommons.org/licenses/by/3.0/). 